\title{
Expérience totalitaire en Europe et poétique du féminin dans Ni poète ni animal d'Irina Teodorescu ${ }^{1}$
}

\author{
Totalitarian experience in Europe and poetics of the \\ feminine in Ni poète ni animal by Irina Teodorescu
}

\author{
Ana Belén Soto \\ Universidad Autónoma de Madrid \\ anabelen.soto@uam.es
}

\begin{abstract}
The main objectif of this article is the critical study of Irina Teodorescu's (Bucarest, 1979) last novel: Ni poète ni animal. This autor is part of a large literary mosaic of intellectuals who, settled in France, have embraced French as their language of adoption and vehicle for literary expression. The novelistic adventure analyze in this paper is framed in that Autre Francophonie (Nowicki \& Mayaux, 2012) linked to the events of 1989. The novel proposal presented here allows us to study the behavior of individuals subject to the requirements of the totalitarianism systems.
\end{abstract}

\section{Key-words}

Irina Teodorescu, women's writing, totalitarianism, deterritorialization, Eastern Europe.

\begin{abstract}
Resumen
El presente artículo se plantea como principal objetivo el estudio crítico de la última novela de Irina Teodorescu (Bucarest, 1979): Ni poète ni animal. Esta autora se enmarca en un amplio mosaico literario de intelectuales que, instalados en Francia, han adoptado el francés como lengua de adopción y vehículo de expresión literaria. La aventura novelesca aquí analizada se enmarca en esa Autre Francophonie (Nowicki \& Mayaux, 2012) ligada a los acontecimientos de 1989. La propuesta novelesca aquí presentada nos permite analizar el comportamiento de los individuos sometidos a las exigencias de los sistemas totalitarios.
\end{abstract}

\section{Palabras clave}

Irina Teodorescu, escritura femenina, totalitarismo, desterritorialización, Europa del Este.

1 Ce travail s'inscrit dans le cadre des objectifs du projet de recherche $\mathrm{I}+\mathrm{D}+\mathrm{I}$ du Ministère espagnol pour l'Éducation et la Science (référence: PID2019-104520GB-I00). 


\section{Introduction}

Hasta hace tan solo tres décadas, la gran mayoría de la humanidad nacía, vivía y moría en unos pocos kilómetros cuadrados, sujeta a un poder masculino absoluto. Ahora, por primera vez desde el origen de los tiempos, los seres humanos tenemos a nuestro alcance una información global y unas capacidades tecnológicas que nos permiten convertirnos en ciudadanos del mundo (Alfaro, Arias \& Gamba, 2019: 7).

Sur fond de globalisation, les propos de Federico Mayor Zaragoza cités ci-dessus mettent en exergue le changement de paradigme sociétal expérimenté au sein des sociétés contemporaines. Le tissu social est, en effet, placé sous le signe des mutations rapides qui ne se limitent pas à la production d'objets à portée technique et technologique et qui, par conséquent, traversent les différentes sphères géopolitiques et socioécocnomiques. Nous pouvons, de ce fait, affirmer que le caractère changeant, muable et instable des structures sociales contemporaines présentent un scénario propice à la liquéfaction des différentes strates. Autrement dit, si l'aspect solide des sociétés représentait la stabilité d'antan, d'après le philosophe Zygmunt Bauman (2003) la modernité se présente sous un état liquide, perméable et donc favorable à l'adaptation des différents contextes et situations au tournant du XXIe siècle. Le choix épistémologique met ainsi en lumière l'une des caractéristiques inhérentes aux sociétés contemporaines qui, tout comme les liquides, “' 'fluyen', 'se derraman', 'se desbordan', 'salpican', 'se vierten', 'se filtran', 'gotean', 'inundan', 'rocían', 'chorrean', ‘manan', ‘exudan’” (Bauman, 2003: 8) avec souplesse et flexibilité pour s'accommoder aux transformations evolutives de notre temps.

Nombreux sont les intellectuels qui s'attardent sur le changement de paradigme sociétal. Nous devons souligner à ce sujet l'apport d'Amin Maalouf, car il fait de ce débat la pierre angulaire de sa création dans son dernier essai intitulé Le naufrage des civilisations (2019). Il expose ainsi dans son prologue:

\footnotetext{
“de mémoire de rose, on n'a jamais vu mourir un jardinier", soupiraient les philosophes français des Lumières en songeant à l'ordre social et à la monarchie de leur propre pays. Aujourd'hui, les roses pensantes que nous sommes vivent de plus en plus longtemps, et les jardiniers meurent. En l'espace d'une vie, on a le temps de voir disparaître des pays, des empires, des peuples, des langues, des civilisations.

L'humanité se métamorphose sous nos yeux (Maalouf, 2019: 11-12).
}

En toile de fond d'une telle réflexion, nous trouvons une thématique riche et complexe qui est devenue l'un des sujets de choix contemporains: penser le carrefour historique du tissage culturel sous une perspective de mouvement réciproque. Le sujet contemporain se trouve ainsi immergé dans un processus de transformation global qui met en avant l'accélération des bouleversements socio-politiques de notre époque. En effet, tout comme le signale Mireille Delmas-Marty (2016: 22) dans son essai intitulé Aux quatre vents du monde. Petit guide de navigation sur l'océan de la mondialisation, 
sur l'océan de la mondialisation, les signaux d'alerte se multiplient à un rythme accéléré depuis le début du XXIe siècle. Après l'apparition d'un terrorisme sans frontières [...] le monde s'est engagé dans une escalade sans fin de mesures et contre mesures, de ripostes et contreripostes [...]. Les crises financières, devenues mondiales, depuis qu'elles atteignent les pays développées (2007-2008), se répètent d'année en année, avec leur cortège de précarité et d'exclusion, même dans une région aussi riche que l'Europe, comme la Grèce peut attester. Simultanément se déclenchent des crises sanitaires.

Naviguer dans des eaux troubles sans pour autant faire naufrage, voici le défi des sociétés contemporaines. Flâner, vadrouiller et déambuler dans le spectacle du monde ne suffissent pas à relever ce défi; pour ce faire, l'observation, l'analyse et la réflexion s'imposent. C'est dans ce contexte que l'écrivain d'origine libanaise s'écrie:

saurons-nous en tirer les leçons avant que ces calamités ne nous frappent de plein fouet? Aurons-nous la force d'âme de nous ressaisir et de redresser le cap avant qu'il ne soit trop tard?

Je veux encore l'espérer. Il serait triste que le paquebot des hommes continue à voguer ainsi vers sa perte, inconscient du danger, persuadé d'être indestructible, comme l'était jadis le Titanic -avant d'aller s'abîmer dans la nuit contre sa fatidique montagne de glace, tandis que l'orchestre jouait Plus près de Toi, Seigneur, et que le champagne coulait à flots (Maalouf, 2019: 329-330).

La pensée maaloufienne introduit, par conséquent, une lueur d'espoir qui invite le sujet à s'arrêter sur les jalons historiques ainsi que sur les macrostructures qui définissent le contemporain dans l'objectif d'apprendre du passé pour mieux surmonter les problèmes sociétaux de nos jours. À ce stade de la réflexion, il convient de signaler la compétence littéraire en termes de projection sociohistorique car "d'innombrables textes [...] montrent que la littérature française [et francophone] traite l'ensemble des problèmes qui taraudent nos sociétés occidentales" (Obergöker, 2011: 10). De ce fait, nombreux sont les critiques littéraires qui, tout comme Tzvetan Todorov dans son essai La littérature en péril (2007), constatent le rôle essentiel que joue la littérature dans le développement sociétal, aussi bien d'un point de vue individuel que collectif. En effet, Tzvetan Todorov (2007: 72-73) déclare avec justesse:

comme la philosophie, comme les sciences humaines, la littérature est pensée et connaissance du monde psychique et social que nous habitons. La réalité que la littérature aspire a comprendre est, tout simplement (mais, en même temps, rien n'est plus complexe), l'expérience humaine. C'est pourquoi on peut dire que Dante ou Cervantès nous apprennent au moins autant sur la condition humaine que les plus grands sociologues et psychologues, et qu'il n'y a pas d'incompatibilité entre le premier savoir et le second. Tel est le "genre commun" de la littérature; mais elle a aussi des "différences spécifiques". 
L'édifice romanesque bâtit dans l'extrême contemporain présente ainsi une mosaïque littéraire où l'expérience vécue s'entrelace avec l'expérience fictionnelle. L'aventure autoficctionnelle devient alors un espace de choix pour bon nombre d'écrivains qui "réticents à écrire des pures et simples fictions [conçoivent l'espace de création littéraire] comme une possibilité de parler d'eux tout en s'inventant, par l'écriture même, une existence nouvelle" (Hubier, 2003: 115). La littérature permet, par conséquent, non seulement de préserver mais aussi de transmettre l'expérience d'un auteur au grand public. C'est dans ce contexte que, dans sa leçon inaugurale du Collège de France, le critique littéraire Antoine Compagnon (2007: 63) mettait l'accent sur la transmission des connaissances et des observations "des autres, ceux qui sont éloignés de nous dans l'espace et le temps, ou qui diffèrent de nous par les conditions de leur vie. [La littérature] nous rend sensibles au fait que les autres sont très divers et que leurs valeurs s'écartent des nôtres". Le champ de création littéraire en langue française présente un scénario propice à l'exposition de la multiforméité environnante et situationnelle à travers une multiplicité de perspectives.

Dans ce contexte, force est de constater le surgissement, au tournant du siècle, d'une génération d'écrivains venus d'ailleurs qui ont choisi le français comme langue et véhicule d'expression littéraire. Le socle de la littérature francophone présente de ce fait une brèche artistique qui témoigne des transformations géopolitiques des dernières décennies. Dans ce vaste archipel de création littéraire nous nous intéressons très spécialement à ces écrivains qui, venus de cette Europe que l'on appelait de l'Est, offrent un espace de création littéraire marqué par les totalitarismes, l'oppression, la résistance et la quête de liberté. L'espace de création devient ainsi "un lieu libre de parole, qui offre à la société la distance nécessaire pour considérer son passé proche. Elle porte aussi un œil critique sur son propre passé, le réalisme socialiste, sans pour autant le nier" (Bressière, 2011: 187). Il s'agit, en effet, d'un corpus d'écrivains dont l'aventure romanesque supprime la mission de guider le peuple prônée autrefois par la littérature communiste et, en même temps, sublime le pouvoir de la littérature. C'est ainsi que, tout comme le signale Antoine Compagnon (2007: 47), nous pouvons déclarer que "la littérature, à la fois symptôme et solution du malaise dans la civilisation" se sert de l'artifice communicatif pour transmettre une expérience fictionnelle empreinte de subjectivité, de romanesque et de poétique.

Nous devons soulever à ce stade de la réflexion la date de 1989 comme un jalon en Europe non seulement d'un point de vue historique, mais aussi du point de vue de la création littéraire. Les systèmes totalitaires, tel que le signale Isabelle Grell (2014: 65), "marquèrent [...] au fer rouge le devoir d'écrire dans la peau. [...] Le 'je' survivant se devait alors d'enclore, d'embrasser les souvenirs, obligé de rendre visible la disparition de l'autre, d'un 'soi', d'un rêve de vie'. Il s'agit, en effet, d'un corpus d'écrivains qui fera usage de 
leur voix non pour faire un "roman historique" sur cette dépossession de leur vie, mais pour dire l'insupportable survivance sur fond d'une expérience traumatique. [...] Il est par exemple évident que dans les pays dits de l'Est, la Serbie, la Pologne où la vérité est prise très au sérieux, voire l'ancienne RDA, les bouleversements politiques ont libéré une écriture du "je" qui se bat pour l'individu, l'individualité, la liberté d'être soi et de manifester ce "moi" trop longuement oppressé dans un groupe imposé par la politique. La prise de parole en son nom propre de Herta Müller, d'origine roumaine, a d'ailleurs été récompensée du Prix Nobel de littérature en 2009 (Grell, 2014: 65-66).

L'année de 1989 est également le moment historique où se situe le dernier roman d'Irina Teodorescu paru en 2019 aux éditions Flammarion et intitulé Ni poète ni animal. Écrivaine d'origine roumaine, Irina Teodorescu illustre un exemple paradigmatique de ces écrivains déterritorialisés qui présentent une démarche volontaire vers la France comme pays d'accueil et vers la langue française comme moyen d'expression littéraire. Ayant vécu les dernières années du communisme dans sa Roumanie natale, l'auteure se sert de son expérience vécue pour donner vie à une aventure romanesque où la petite histoire se trouve intimement liée à la grande Histoire. C'est pourquoi, nous nous proposons d'explorer dans cet article les enjeux inscrits dans cette année charnière en Europe qui, de plus, symbolise l'ancrage de l'édifice fictionnel ici analysé. Par la suite, il convient d'ébaucher l'expérience totalitaire au sein des xénographies francophones de l'extrême contemporain à travers la poétique du féminin. Il reste, dès lors, à s'interroger sur la mise en scène de l'expérience totalitaire comme artifice communicatif dans Ni poète ni animal. Pour ce faire, nous nous attarderons sur les modèles féminins évoqués au cœur du roman pour esquisser, en outre, les lieux de représentation du système dans cette année 1989. Cette réflexion, qui prône une vision sociale humaniste, nous permettra de mieux comprendre les enjeux sociétaux intrinsèquement liés aux totalitarismes en Europe à travers un roman protéiforme inscrit dans l'extrême contemporain.

\section{1989, un jalon dans l'histoire}

Dans le panorama international, 1989 s'érige en symbole d'un changement de paradigme en Europe, mais aussi dans le monde. D'après l'historien Jean-François Soulet (2006: 132) la chute du mur de Berlin et, par conséquent, des systèmes totalitaires sous le signe du communisme

a surpris le monde entier par sa soudaineté et sa rapidité. Jacques Rupnik rappelle que les étudiants manifestant à la fin de novembre 1989 dans les rues de Prague avaient inscrit sur une banderole: "Pologne, 10 ans; Hongrie, 10 mois; RDA, 10 semaines; Tchécoslovaquie, 10 jours"; formule que l'on pourrait aujourd'hui compléter par "Bulgarie, 1 jour; Roumanie, 10 heures". À elle seule, elle résume le processus en chaîne des mutations et son accélération vertigineuse à compter de juillet 1989 . 
Il s'agit, en effet, d'une évolution socio-historique qui reflète l'hétérogénéité de cette Europe que l'on appelait de l'Est. L'écosystème multiple et pluriel de ce vaste territoire vivant sous un même système politique a provoqué, en conséquence, une réponse multiforme aux situations et aux événements datés de 1989. C'est dans ce contexte que les chercheurs Jerôme Heurtaux et Cédric Pellen signalent dans leur introduction à l'ouvrage collectif intitulé 1989 à l'Est de l'Europe. Une mémoire controversée (2009: 6):

\begin{abstract}
il n’y a pas "d'événement 1989" ou plutôt il y a autant "d'événements 1989" qu'il y a de versions nationales de la sortie du communisme. D'un pays à l'autre, ce ne sont ni la même temporalité, ni les mêmes faits saillants, ni les mêmes dimensions matérielles dont les traces s'objectivent dans le récit national. Ces variations se retrouvent dans les qualifications multiples de la sortie du communisme: le "tournant" allemand ou la "transition" hongroise ne font pas appel au même lexique du souvenir que la "Révolution" roumaine. Ainsi, parler de "l'événement 1989" revient à unifier artificiellement plusieurs 1989 sous une symbolique commune.
\end{abstract}

Si l'évolution politique sous le gouvernement de Gorbachev a présenté un scénario favorable aux transformations géopolitiques, socioéconomiques et historiques inhérentes aux événements de 1989, le succès d'une telle entreprise se trouve dans le mécontentement de la société civile. C'est ainsi que l'historien Henry Bogdan (1990: 635) convoque "les jeunes, ouvriers et intellectuels réunis dans un même désir de liberté" à l'origine des bouleversements. Il affirme, de même, que "ce sont eux qui, par leurs manifestations pacifiques, ont fait reculer le pouvoir communiste et imposé la démocratie. Pourtant, ils n'avaient jamais rien connu d'autre que le système dans lequel ils vivaient. Pour la propagande officielle, ils étaient l'avenir du socialisme" (Bogdan, 1990: 635). L'historien polonais Karol Modzlewski (Hertaux \& Perren, 2009: 296), quant à lui, réclame le mauvais fonctionnement du système communiste comme source d'effondrement. De même, les agents internationaux ont joué un rôle important dans cette évolution. Nous devons souligner à ce sujet la politique internationale des États-Unis ainsi que du Vatican. La tactique reaganienne, d'une part, visait la Pologne comme le maillon faible du système et dessinait son hypothèse comme suit: "si nous pouvions arriver à secouer et à déstabiliser la Pologne alors les ondes de choc irradieraient dans toutes les directions: vers l'Ukraine et les Balkans, en Lettonie, en Lituanie et en Estonie" (Soulet, 2006: 137). D'autre part, le Vatican s'était également prononcé en Pologne dès 1979, lors de la première visite du Pape Jean-Paul II. C'est pourquoi Jean-François Soulet (2006: 136) soutient que

sans reprendre à notre compte la thèse de l'entente (ou du complot) qui, selon le journaliste américain Carl Bernstein aurait été scellée au Vatican, en juin 1982, entre Ronald Reagan et Jean-Paul II, dans le but de renverser le communisme, il est indéniable que les deux hommes exercèrent, chacun à leur manière, une influence majeure sur l'évolution de la Pologne durant toute la décennie quatre-vingt. 
Il s'agit, néanmoins, d'un processus complexe qui a suivi des transformations différentes dans chaque pays de l'ancien bloc soviétique. Force est de constater à ce stade de la réflexion que l'héritage soviétique a fait partie du processus de construction d'une mémoire collective où "le régime communiste n'est pas seulement perçu comme une période de répression; il y a été aussi une période de modernisation de la société et de l'État [...]. Deux modes d'interprétation de ce passé cohabitent dans la conscience collective" (Soulet, 2006: 217). C'est ainsi que dans le processus kaléidoscopique d'adaptation aux nouveaux enjeux sociétaux, les pays d'antan satellites de l'ancienne URSS vont illustrer une multiplicité de spécificités à la fois qu'ils convergent dans le processus de démocratisation et d'adaptation à l'économie du marché. Cette évolution implique un changement des macrostructures politiques et économiques qui s'avère être long et complexe, mais aussi paradoxal. En effet, si le processus né d'une prise de conscience et de parole de la diversité inhérente aux différentes ex-democraties populaires, le processus "ha desembocado en la uniformidad. Libres de los yugos politicos que las uncieron, las naciones de la Europa centro-oriental [...] se han convertido en [...] aspirantes a ciudadanos de la aldea-mundo gobernada por el sistema capitalista" (Martínez de Sas, 1999: 82).

À ce stade de la réflexion, et en guise de conclusion à cette synthèse historique, il convient de s'attarder sur les effets des événements de 1989 dans cette Europe que nous appelions de l'Est. Suite à la chute du mur de Berlin, une nouvelle géopolitique s'est dessinée à l'Est de l'Europe avec de nouvelles lois et de nouvelles réalités, certains pays même avec de nouveaux noms, ce qui implique une évolution identitaire des sociétés vivant ces bouleversements. Nombreuses sont, en effet, les questions qui se posent dans ce contexte. Nous devons souligner deux questions intimement liées à l'expérience littéraire de ces écrivains appartenant aux xénographies francophones de l'extrême contemporain. D'une part, comment interpréter le choix d'un pays comme terre d'accueil et d'une langue, autre que la maternelle, comme véhicule d'expression littéraire pour ces individus? Et, d'autre part, pourrions-nous affirmer que les citoyens de cette Europe autrefois appelée de l'Est se sentent plus proches de l'interculturalité suite au démembrement de l'URSS? Loin d'avoir une réponse universelle, nous nous permettons de parler et de faire parler ces voix littéraires qui mettent en lumière, à travers l'expérience vécue à la première personne, un tissu fictionnel perméable aux événements de l'Histoire.

\section{L'expérience littéraire au sein des xénographies francophones au féminin}

La littérature contemporaine d'expression française témoigne du surgissement d'un espace littéraire transnational qui rompt non seulement avec les canons traditionnels mais aussi avec des conceptions idéologiques enracinées dans des systèmes souvent obsolètes qu'elle se propose de mettre en perspective. Par l'écriture, il s'agit de présenter une nouvelle réalité qui se forge au sein même d'une société désormais plurielle et qui s'inscrit dans un monde global. Un nouveau paradigme se profile ainsi dans une 
perspective dynamique et transversale. Il s'agit d'une littérature ectopique (Albadalejo, 2011), écrite hors lieu et caractérisée par la construction d'une identité personnelle en rapport avec un projet existentiel qui peut renvoyer à une notion plurielle d'ailleurs (Alfaro, Sawas \& Soto, 2020: 9-10).

La littérature francophone contemporaine présente, en effet, depuis les deux dernières décennies une mosaïque artistique qui relève l'essor d'un nouveau paradigme littéraire et qui met en lumière les voix de l'ailleurs. Si la littérature francophone était autrefois intimement associée aux phénomènes de colonisation et de post-colonisation, cette affirmation n'est plus de mise. Nombreux sont les intellectuels appartenant à cette Autre Francophonie analysée par Catherine Mayaux et Joanna Nowicki (2012: 9) qui avait été “marginalisée, souvent occultée au profit de la francophonie du Sud ou d'autres francophonies mieux reconnues dans une géopolitique mondiale". Il est intéressant de s'attarder sur l'apport de ces intellectuels venus de cette Europe que nous appelions de l'Est car "bien des écrivains et intellectuels venus de ces pays s'en sont nourris, sont redevables des apports de la culture française à leur propre culture et création et ont enrichi celle-ci en retour dans un travail d'échange mal connu ou reconnu" (Nowicki \& Mayaux, 2012: 9).

Alors, pourquoi choisir la France comme terre d'accueil et le français comme véhicule d'expression littéraire pourrions-nous nous demander? Pour ces intellectuels venus de l'autre côté du rideau de fer, ce choix est fort symbolique, car ils cherchent "la liberté perdue dans leur pays d'origine, liberté d'opinion, liberté d'expression, liberté de conscience, et parfois la liberté d'être soi-même et non pas l'enfant de sa patrie" (Nowicki \& Mayaux, 2012: 119-120). La France acquiert ainsi un rôle de mythe fondateur sans doute influencé par l'image internationale qu'elle projette à travers sa triade Liberté, Égalité, Fraternité, mais aussi comme socle des Droits de l'Homme, de cosmopolitisme et même de glamour. La langue française, quant à elle, devient une sorte de refuge pour certains de ces auteurs ainsi qu'introduit une "forme de dissidence" (Nowicki \& Mayaux, 2012: 151). L'acquisition de la langue française se rend, alors, indispensable non seulement dans l'objectif de la réussite du processus d'intégration dans la société d'accueil, mais aussi comme moyen de transmettre une réalité vécue dans une autre langue. C'est dans ce contexte que certains auteurs, tels que Rouja Lazarova, mettent en question leur propre rapport à la langue d'origine. Dans son premier roman, intitulé Sur le bout de la langue, ce questionnement se trouve au cœur de la réflexion non seulement tout au long de 19 chapitres intitulés à partir des expressions lexifigées en langue française, mais aussi dans l'acte de clôture romanesque où la réflexion métalitteraire s'impose:

un seul regret hante mon existence. J'aimerais écrire ce livre sur l'apprentissage d'une langue; je ne pourrais cependant le traduire en ma langue maternelle. Peut-on concevoir l'existence d'un texte intraduisible? Peut-on penser un manuscrit qui ne serait pas lu par les lecteurs auxquels il se destine?

Vais-je écrire un jour, ce livre, ou me restera-t-il sur le bout de la langue? (Lazarova, 1998: 142). 
Le champ littéraire francophone s'est ainsi élargit et enrichit grâce à l'apport de ces intellectuels qui, immigrés ou exilés, embrassent la langue française et parsèment les étals des librairies. Force est de constater l'intérêt qu'un groupe réduit de chercheurs porte au sujet de cette lézarde dans le socle littéraire francophone. C'est dans ce contexte que nous devons souligner la contribution scientifique d'Anne-Rosine Delbart qui présente en 2005, sous le titre Les exilés du langage. Un siècle d'écrivains français venus d'ailleurs (1919-2000), un bilan sur le vaste archipel littéraire écrit en langue française. Véronique Porra, par la suite, s'attardera également sur les écrivains allophones d'expression française dans son ouvrage intitulé Langue française, langue d'adoption. Une littérature "invitée”, entre création, stratégies et contraintes (1946-2000), et publié en 2011. Le tournant du siècle devient ainsi la marge temporaire qui permet la réflexion sur le développement de l'activité littéraire dans le contexte qui nous occupe. Ce sera en 2012 lors de la parution de Passages et ancrages. Dictionnaire des écrivains migrants de la langue française (1981-2011) dirigé par Ursula Mathis-Möser et Birgitz Mertz-Baumgartner que nous verrons paraître le nom des écrivains publiés après l'an 2000. Il est intéressant de se pencher également sur l'ouvrage dirigé par Joanna Nowicki et Cahterine Mayaux L'Autre Francophonie car, publié également en 2012, il présente une focalisation nouvelle sur ce champ de création littéraire. Il s'agit de parler et de faire parler de cette autre francophonie, ancrée dans le continent européen et issue de cette Europe autrefois appelée de l'Est.

En outre, suivant l'esprit de l'ouvrage que nous venons d'évoquer, Évelyne Enderlein et Lidiya Mihova publient en 2013 Écrire ailleurs au féminin dans le monde slave au XXe siècle. L'analyse de l'ectopos sous le regard des femmes au sein de la production littéraire provoque ainsi une réflexion sur l'aspect sexué en termes de création. Il est évident que le rôle de la femme dans les sociétés contemporaines est devenu l'un des enjeux sociétaux au niveau global. C'est dans ce contexte que nous devons, de même, souligner la récente parution de Xénographies féminines dans l'Europe d'aujourd'hui (Alfaro, Sawas \& Soto, 2020), car cet ouvrage collectif se penche également sur l'apport féminin dans le socle littéraire. La pierre angulaire de cet ouvrage est ainsi présentée sous une double perspective. Dans un premier temps, il s'agit de mettre en lumière le terme de xénographie pour désigner "une vaste constellation de situations liées à l'immigration, à l'exil et au voyage volontaire, dont la caractéristique principale est la rencontre avec l'altérité sous différentes manifestations (linguistiques, sociales, culturelles, artistiques et idéologiques)" (Alfaro, Sawas \& Soto, 2020: 10). Puis, la perspective féminine permet d'aborder l'apport littéraire de ces femmes venues d'ailleurs qui acquièrent un rôle de plus en plus visible dans le panorama littéraire de l'extrême contemporain.

À la lumière de ce brassage terminologique, force est de constater notre préférence actuelle pour le concept de xénographie francophone qui met en lumière non seulement la mosaïque littéraire en langue française, mais aussi la multiplicité et la multiforméité cultu- 
relle et identitaire transmises dans la langue d'accueil. L'importance accordée à l'écriture au féminin est également un aspect que nous partageons avec la perspective d'analyse présentée dans les ouvrages cités ci-dessus, car tout comme le signalent Évelyne Enderlein et Lidiya Mihova (2013: 15) "les études sur la littérature de l'émigration restent, la plupart du temps, muettes sur les plumes féminines, nombreuses pourtant, mais laissées dans l'ombre par le dictat du canon littéraire et, souvent politique".

Nombreux sont, en effet, les écrits féminins qui tapissent les rayons des librairies. Agota Kristof (1935-2011) d'origine hongroise, Oana Orlea (1936-2014) d'origine roumaine, Luba Jurguenson (1958-) née à Moscou dans une famille d'origine estonienne ou encore Rouja Lazarova (1968-) d'origine bulgare représentent quatre de nombreux exemples paradigmatiques de ces plumes féminines qui parsèment le panorama littéraire des xénongraphies francophones de l'extrême contemporain. L'expérience romanesque de ce corpus d'écrivaines fortement influencées par les événements de 1989 met en exergue, de plus, l'émergence d'un personnage conçu au féminin, présenté dans son quotidien et écarté des stéréotypes. L'expérience totalitaire et la transition démocratique s'érigent, également, en toile de fond d'une telle représentation qui permet d'aborder une multiplicité d'expériences multiformes et plurivoques permettant de penser le sujet ayant vécu l'expérience totalitaire sous un regard de femme.

La création littéraire au féminin présente ainsi une perspective qui diffère de la manière dont les hommes sentent, interprètent et représentent l'expérience vécue. En effet, tout comme le signale la spécialiste Christine Planté (2003: 658), le genre du regard évoque une différence essentielle dans 'l'expérience humaine et la vision qu'on a du monde et de la culture n'est pas la même selon que les femmes y apparaissent seulement en position d'objets, d'inspiratrices ou de lectrices, ou si elles figurent aussi en position de productrices et créatrices". La prolifération des textes écrits par des femmes permet également de mettre en question les canons esthétiques littéraires ainsi que de s'interroger sur les nouvelles approches des figures féminines dans le tissu fictionnel. Dans ce contexte, la littérature expose l'importance accordée à la première personne et le choix thématique intimement lié aux récits d'enfance ou d'adolescence, aux histoires familiales et aux souvenirs sous un système totalitaire. En effet,

les bouleversements politiques après 1989 marquent une autre étape dans la recherche et dans la construction d'une nouvelle identité culturelle et artistique. La première décennie qui suivit les changements est marquée par le foisonnement d'ouvrages littéraires qui réfléchissent sur le totalitarisme et le dénoncent. [...] Les années 1990 se caractérisent par l'apparition d'une nouvelle génération de créateurs "postmodernistes" qui se lancent dans les expérimentations pour déplacer et pour ébranler les limites des genres, pour multiplier les points de vue ou bien pour déconstruire les modèles littéraires connus (Enderlein \& Mihova, 2013: 18). 
Les xénographies francophones de l'extrême contemporain offrent ainsi une pluralité de regards sur la constellation soviétique à partir de l'expérience vécue. C'est dans ce contexte que nous devons souligner l'apport todorovien en matière de réflexion identitaire, comportementale et cognitive à travers la réflexion évoquée notamment dans ses essais Face à l'extrême (1991), L'homme dépaysé (1996) et L'expérience totalitaire (2009). D'après Tzvetan Todorov (2009: 41) "la connaissance du passé est indispensable à l'action dans le présent, et en même temps elle lui reste subordonnée”. En effet, pour mieux appréhender le monde qui nous entoure il faut s'attarder sur les passages et ancrages des événements passés qui forgent le socle des sociétés contemporaines, sans pour autant stigmatiser les épisodes suivant la conception binaire qui traditionnellement oppose le bien et le mal. D'après Tzvetan Todorov (2009: 309), il faut s'interroger sur les conditions qui ont provoqué de tels événements car même si "on ne parviendra jamais à délivrer les êtres humains du mal. Notre seul espoir est, non de l'éradiquer définitivement, mais de tenter de le comprendre, le contenir, l'apprivoiser". À cet égard, il est intéressant de comprendre ce qui représente le mal dans le discours todorovien où le mal désigne "un terme commode recouvrant une série de violences fort variées: guerres, génocides, massacres, tortures, viols, crimes, souffrances infligées et subies" (Todorov, 2009: 284) et le bien serait donc une sorte d'absence d'hostilité. La transmission de l'expérience totalitaire devient alors dans ce contexte un devoir mémoriel car, même si les régimes totalitaires ne représentent pas une forte menace dans l'Europe d'aujourd'hui, leur empreinte est encore vivante et le construit social doit encore tirer des enseignements sur notre passé récent. C'est par ailleurs dans ce sens que l'intellectuel d'origine bulgare souligne pertinemment:

la vie sous le totalitarisme a été comme une expérience conduite à ciel ouvert, dans l'immense laboratoire constitué par plusieurs pays d'Europe et d'Asie. Par similitude ou par contraste, elle permet d'éclairer aussi la vie des habitants des pays démocratiques. Maintenir en vie ces leçons fait partie de nos devoirs d'aujourd'hui (Todorov, 2009: 15).

C'est alors que nous pouvons affirmer que l'apport de ces auteures venues de l'Est et du Centre du continent européen contribue, par conséquent, à une réflexion aussi bien sur les empreintes totalitaires dans le sujet contemporain que sur le rôle de la femme dans les sociétés de nos jours. Cette réflexion nous permet de penser

la francophonie, les autres francophonies, la sphère francophone [comme] de leviers pour construire une communication entre les peuples que tout semble à priori opposer. La francophonie illustre le sens profond de la communication: moins partager qu'apprendre à cohabiter (Nowicki \& Mayaux, 2012: 329).

La littérature embrasse donc les enjeux sociétaux de notre temps non seulement pour évoquer mais aussi pour comprendre un espace géopolitique marqué par une multiplicité 
discursive à savoir l'Europe de l'Est, l'Est de l'Europe, l'Europe Médiane, Mittleeuropa, les Balkants, parmi d'autres exemples. Autant de définitions que de manières de voir et d'appréhender l'Histoire d'un continent autrefois divisé. L'archipel littéraire des xénographies francophones nous permet, en conséquence, de constater la territorialité de l'Europe dans un contexte géopolitique où se dessine une nouvelle géopoétique à travers les plumes de ces écrivaines venues d'ailleurs.

\section{Ni poète ni animal}

C'est dans ce contexte que nous nous permettons d'analyser dans notre article $\mathrm{Ni}$ poète ni animal le dernier roman d'Irina Teodorescu. Née à Bucarest le 30 mars 1979, Irina Teodorescu, tout comme son alter-ego fictionnel (Varier, 2020: en ligne), n'a que 10 ans lors de la "Révolution" de décembre 1989. Installée à Paris depuis 1998, elle est l'auteure de quatre romans, un récit et un recueil de nouvelles. De même, ayant étudié les Beaux Arts dans sa Roumanie natale, elle est graphiste et elle expose régulièrement ses pièces d'art. C'est par ailleurs dans cette perspective que l'artiste prépare, en ce moment, un roman qui imbrique les arts plastiques et la littérature et dont le sujet est le corps féminin. Nous devons souligner, en outre, que cette auteure, très vite remarquée par la critique dès la parution de son premier roman (La malédiction du bandit moustachu, 2014), a obtenu plusieurs distinctions telles que le prix Récit de l'Ailleurs pour Les étrangères en 2017. Elle a également bénéficié, pour l'écriture du roman objet de cet article, d'une résidence à la Villa Marguerite Yourcenar ainsi que d'une bourse du Centre National du Livre. Nous pouvons affirmer, de ce fait, que cette auteure représente un exemple paradigmatique de ce corpus d'écrivaines dessinant le parcours des xénographies francophones dans le contexte européen de l'extrême contemporain.

Ni poète ni animal est un roman dont l'espace prétextuel se situe dans cette Roumanie au tournant des années quatre-vingt-dix où le contexte politique et le mécontentement populaire ont donné suite à l'un des événements de 1989: la "Révolution" de décembre 1989. C'est ainsi que l'espace textuel situe une jeune fille de dix ans, appelée Carmen, au centre d'un noyau thématique où, sous le regard de l'enfance, l'auteure évoque l'emprise totalitaire autour des événements de cette année charnière. Le discours de ce personnage est entrecoupé par la transcription des enregistrements de sa mère, ainsi que par des extraits de conversation dans l'hôpital où sa grand-mère maniaco-dépressive avait été internée cette année-là. La voix de cette jeune fille devenue adulte se prononce également dans l'espace textuel qui, divisé en quatre structures discursives, présente un métadiscours imbriquant la petite histoire et la grande Histoire dans l'expérience littéraire. C'est, par ailleurs, la mort soudaine de l'ami de la protagoniste, le grand poète, qui joue l'acte inaugural du discours romanesque. 
Le lecteur se trouve ainsi immergé dans une histoire individuelle inscrite dans l'Histoire collective où la structure psychosensorielle du roman illustre l'importance accordée à l'expérience autofictionnelle. Irina Teodorescu, qui avait déjà traité le sujet des totalitarismes aussi bien dans Les étrangères que dans Celui qui comptait être heureux longtemps, fait de l'aventure romanesque dans Ni poète ni animal la plus intime. La langue française devient ainsi pour l'auteure non seulement un véhicule de l'expression littéraire, mais aussi le moyen d'appréhender les empreintes totalitaires dans le sujet contemporain. C'est ainsi qu'Irina Teodorescu dépoussière les souvenirs de sa mémoire à la fois qu'elle entame un processus d'information nécessaire à la compréhension des épisodes ancrés dans sa mémoire lorsqu'elle avait dix ans (Varier, 2020: en ligne). L'auteure fait ainsi partie de ces intellectuels qui affirment le devoir de témoigner (Todorov, 2004: 16) parce que, le totalitarisme n'est pas pour ces écrivains "un thème parmi d'autres, choisit en raison [d'une] curiosité intellectuelle; il correspond à une expérience vécue qui a joué un rôle central pendant la première partie de [leur] existence" (Todorov, 2009: 10). L'écriture de Ni poète ni animal pourrait alors se caractériser comme un voyage initiatique où, mot à mot, la langue de l'autre construit le tissu autofictionel.

C'est dans ce contexte que nous nous attardons sur les modèles féminins évoqués au cœur du roman pour esquisser l'expérience vécue sous le joug totalitaire ici tracée. Le regard féminin, romancé à partir de trois génération de femmes d'une même lignée, nous permet non seulement de nous pencher sur le rôle de la femme, mais aussi sur l'espace référentiel de l'entreprise totalitaire. Carmen, Ema et Dani évoquent ainsi un parcours intergénérationnel où le lecteur découvre trois perspectives différentes d'une même expérience. C'est ainsi que, dans l'objectif de mettre en avant l'empreinte des totalitarismes, l'auteure s'éloigne des descriptions physiques des personnages pour s'attarder sur les aspects psychosensoriels de l'expérience totalitaire.

Carmen, alter-ego de l'auteure, est un personnage qui se trouve dédoublé dans son discours. Dans un premier temps, c'est la femme adulte qui prend la parole pour mettre en musique son étrangeité (Kristeva, 1988). Elle dira, en effet, dans le premier paragraphe du roman "il y a un mot dans ma langue natale pour désigner les arbres auxquels je pense" (Teodorescu, 2019: 7). La toile de fond de la représentation est ainsi levée sur un personnage étranger qui, installé à Paris, apprend par les journaux la mort de son ami resté anonyme tout au long du roman, mais caractérisé par son rapport à la littérature et à l'ancrage identitaire. C'est, en effet, "le grand poète aux beaux yeux et aux cils incurvés" (Teodorescu, 2019: 67), qui sera également nommé comme "Ma Terre Glissante Que J'ai Fuie" (Teodorescu, 2019: 123). Puis, dans le chapitre suivant, le discours projeté sous le prisme de l'enfance se construit sous une forme de journal intime présenté par mois. Le mois de mars est donc le mois qui ouvre ce processus mémoriel où la protagoniste se présente comme suit: 
je m'appelle Carmen. J'ai aujourd'hui deux nationalités, deux pays donc, deux pièces dans l'appartement où je vis seule la grande majorité du temps. Je suis née le ler avril à l'étranger. J'exerce le métier d'avocate et partage un cabinet rue Lafayette, à Paris, avec deux confrères, nous sommes des généralistes du droit civil, des auxiliaires de la justice ordinaire (Teodorescu, 2019: 14).

L'imbrication de l'identité d'origine et de l'identité d'accueil représente ainsi la clé des processus d'intégration et d'évolution identitaire. Il est intéressant de souligner à ce stade de la réflexion comment Carmen -personnage- et Irina -auteure- font de leur double appartenance leur étendard identitaire. Carmen, cependant, reste un personnage quelque peu mystérieux pour son entourage français car la toile de la représentation se termine comme suit: "et moi, poétesse myope de CM2, avocate à l'étranger, fugue d'un grand poète, amie d'un cochon, sparadrap d'une grand-mère folle, j'arrache mes masques" (Teodorescu, 2019: 211). Le lecteur se trouve ainsi confronté à l'évolution d'un personnage qui dans un premier temps voyait dans la révolution de son autre pays, "un moment de grâce, [...] [où] le temps des dictateurs était terminé et [...] commençait le règne des poètes" (Teodorescu, 2019: 9). La révolution est alors évoquée sous un prisme idéalisé où les poètes symbolisent la liberté d'expression. La suite de cette révolution se dessine, cependant, sous le signe du "quartier général [d'un] libéralisme sauvage" (Teodorescu, 2019: 72).

La littérature, de la main des poètes, s'érige ainsi en étendard de liberté, de dissidence et de démocratie. La langue est ainsi mise à l'honneur non seulement dans la construction identitaire des identités multiples, mais aussi comme expérience littéraire. C'est n'est donc pas un hasard si la protagoniste commence à écrire des poèmes depuis son plus jeune âge et fait de l'école l'un des espaces référentiels des épisodes consacrés à l'enfance. Suivant les consignes gouvernementales, toutes les écoles devaient présenter une structure similaire dans les salles classe. C'est dans ce contexte que la petite Camen décrit son espace scolaire comme suit:

il y avait dans notre salle de classe, comme dans toutes celles du pays, accroché au-dessus du tableau, précisément au milieu, le portrait du président de notre République jeune, de trois quarts, souriant et très ressemblant, du moins de mon point de vue, au camarade maître du CM2 C. Pour me faire une image plus précise du visage de ce fameux Parti, je composai dans ma tête un joyeux mélange des deux. Je pondis sans difficulté une dizaine de strophes dans lesquelles je chantais sans vergogne ses louanges, d'abord en tant que père - le Parti avait remplacé Dieu et était, on nous l'avait assez martelé, notre père à nous-, ensuite en tant que mari -certainement qu'il avait une femme, vu le nombre de ses enfants-, puis en tant que maître d'école et, enfin, je m'avançais un peu dans le dernier quatrain, en tant que vieux et sage grand-père (Teodorescu, 2019: 68-69).

Virtuose de la langue poétique acceptée par le Parti, la jeune Carmen écrit de poèmes qui seront, par la suite, lus en cours. En même temps, elle présente sous le seuil naïf de son 
jeune âge une réalité qui sera beaucoup plus contestée par d'autres personnages qui, tout comme ses parents “ne s'étaient jamais présentés devant [la camarade maîtresse] avec les cadeaux requis en vue d'une amélioration indéniable de [sa] moyenne. Pourtant c'était une chose courante" (Teodorescu, 2019: 70). Le message réfractaire passe ainsi sous le filtre de l'humour et de l'innocence traditionnellement accordé à l'enfance. Le regard de Carmen permet ainsi de visionner en l'espace d'un roman les différentes situations parfois même paradoxales dans le rapport interpersonnel sous l'emprise totalitaire. À ce stade de la réflexion, force est de souligner l'épisode de la dispute de ses grands-parents paternels avec ses voisins au sujet d'un cerisier. Des années plus tard, la femme adulte prend la parole pour s'écrier:

la dispute de mes grands-parents avec leurs voisins demeure pour moi incompréhensible, et le mystère s'est accru pendant mes études de droit. Comment dans une république socialiste prônant les valeurs du partage, de la collectivité, de la fraternité et de l'égalité, une femme que j'aimais profondément [...] et cette autre femme avec qui j'avais eu d'agréables conversations [...] avaient réussi à se mépriser à un tel point et à se jeter au visage de telles accusations? Manque de générosité, avarice, parasitisme, avidité, et même vol à la sauvette -juste pour un petit bol, une fois, pour faire une mousse (Teodorescu, 2019: 89).

C'est ainsi que la jeune Carmen est représentée au cœur de l'histoire dans des espaces clos tels que l'école ou les maisons familiales. Ces espaces référentiels situent la protagoniste dans la dualité créée entre l'espace public et l'espace privé, entre le discours public et le discours privé. En effet, sous les totalitarismes il existe un discours officiel, représenté dans l'édifice romanesque par les poèmes écrits par la jeune Carmen, et d'un discours privé que les individus utilisent "chez [eux], entre amis, ou pour tout domaine que l'idéologie ne touche pas trop près, tel le sport ou la pêche" (Todorov, 1996: 42). Les enfants, ne pouvant pas contrôler cette schizophrénie discursive, étaient en général exclus de tout discours relevant du domaine privé tout comme le signale l'auteure dans son interview avec Zoé Varier pour France Inter (2020). C'est ainsi que sa mère, Ema, doit se cacher dans la salle de bains pour enregistrer dans un dictatophone des propos parfois subversifs contre le système.

La salle de bains représente pour Ema un espace privé où elle peut vaincre la schizophrénie discursive imposée par le système. Dans cet espace clos où elle se sent en sécurité, cette mère enregistre des cassettes pour se décharger et partager son expérience "deux fois par semaine pendant dix ans [...] le temps d'une face, soit une demi-heure" (Teodorescu, 2019: 36). La salle de bains symbolise de ce fait une salle de consultation et l'enregistrement une thérapie que l'auteure partage avec le lecteur sous forme de six chapitres intitulés en fonction du numéro et de la face de ces "K7". Il est intéressant de s'attarder sur cet espace référentiel car, d'après nos lectures, il s'agit d'un leitmotiv dans l'édifice narratif de bon nombre des auteurs qui, tout comme Irina Teodorescu, se penchent sur l'empreinte totalitaire. Ema est décrite, à travers ses enregistrements, comme une femme forte capable de 
mener à bien ses différents projets. C'est dans ce contexte que nous devons signaler le rapport qu'elle entretient avec sa mère, qui par ailleurs fait une tentative de suicide au moment où se déroule l'histoire. Le lien familial unissant Ema et Dani est fort et, de ce fait, Ema fait de son mieux pour rapprocher sa fille de sa mère. Il s'agit, cependant, d'une relation forte en sensations car même si la figure de la mère est représentée comme une figure unique et bien aimée, les rapports entretenus entre Dani et Ema sont complexes. L'auteure se sert ainsi de l'édifice narratif pour mettre en exergue la multiplicité de rapports existant entre mère et fille. C'est ainsi que si "desde siempre se ha intentado ennoblecer ese binomio en un principio de rivalidad territorial en el seno de una familia hasta depurarlo de toda tensión" (Diego \& Vázquez, 2002: 20), cette affirmation n'est pas de mise dans le texte que nous analysons. La mère de Ema, en effet, loin de représenter ce modèle parfois même idéalisé, est ici dessinée comme un personnage difficile, atteint d'une maladie mentale et "détestable" (Teodorescu, 2019: 25) d'après sa petite fille.

La maladie mentale de Dani évoque dans un premier temps l'accès restreint aux cures sous la période communiste, car les meilleurs espaces sanitaires étaient "réservées à la nomenclature" (Teodorescu, 2019: 30). Ema réussit, cependant, à faire admettre sa mère dans un sanatorium après son passage à l'hôpital Numéro Neuf, "le grand, le principal et peut-être même l'unique hôpital psychiatrique du pays, ou en tout cas de la région sud" (Teodorescu, 2019: 12). Il s'agit d'un personnage fort intéressant non seulement au sujet de son état psychologique mais aussi parce que Dani "était un sujet d'intérêt pour les services secrets communistes, certainement à cause de son héritage familial" (Teodorescu, 2019: 44). Cette affirmation éclos suite à la découverte de Carmen "dans les archives nationales [de] ce gros dossier - [de] dix-sept centimètres d'épaisseur" (Teodorescu, 2019: 44). Ces documents concernant la vie de cette femme s'inscrivent dans le tissu fictionnel lors de trois chapitres où Dani parle avec des médecins spécialistes en santé mentale. L'expérience vécue de cette grand-mère, incomprise de sa petite fille, s'expose ainsi sous une perspective frontalière qui nous permet de penser le sujet de maladies mentales sous les systèmes totalitaires.

Si d'après Érasme (cité par Salah, 2017: 91) "la folie est une ivresse sans fin, où la joie, les délices, les enchantements se renouvellent sans cesse", pour la Ema l'état mental de sa mère représente un véritable soucis. En effet, force est de constater que l'approche pathologique de la santé mentale en rapport avec l'empreinte des totalitarismes présente un double isolement: l'isolement spatial et l'isolement discursif. Comment déterminer alors le degré de cette pathologie chez les individus qui, tout comme Dani, vivent sous les systèmes totalitaires et sont objet d'intérêt pour le gouvernement? Pourquoi Ema se résistait à faire interner sa mère dans l'hôpital Numéro Neuf? Loin d'avoir une réponse concrète, nous pouvons reprendre la réflexion d'Anna Arendt (2002: 306) au sujet de l'isolement: 
tandis que l'isolement intéresse uniquement le domaine politique de la vie, la désolation intéresse la vie humaine dans sa totalité. Le régime totalitaire comme toutes les tyrannies ne pourrait certainement pas exister sans détruire le domaine public de la vie, c'est-à-dire sans détruire, en isolant les hommes, leurs capacités politiques. Mais la domination totalitaire, comme forme de gouvernement, est nouvelle en ce qu'elle ne se contente pas de cet isolement et détruit également la vie privée. Elle se fonde sur la désolation, sur l'expérience d'absolue de non appartenance au monde, qui est l'une des expériences les plus radicales et les plus désespérées de l'homme.

Dani est, en effet, isolée non seulement de son entourage mais aussi du monde. C'est un personnage qui s'intègre dans le roman suite à une tentative de suicide et disparaît à la fin du roman sans pour autant donner d'explications. Il est fort intéressant de souligner le manque d'ancrage ainsi que le déracinement de ce personnage qui n'hésite pas à affirmer dans le sanatorium que sa fille "se donne toujours beaucoup de mal pour [la] placer le plus loin d'elle et le plus long-temps possible" (Teodorescu, 2019: 99). Cette distanciation avec sa fille évoque l'incompréhension maternelle envers Ema qui a non seulement des obligations professionnelles, mais aussi familiales. Ema devient ainsi un personnage de l'entre-deux qui représente aussi bien la mère que la fille, elle se démène pour bien assouvir les besoins de sa mère et de sa fille, à la fois qu'elle doit, par exemple, garder son poste car "le travail est en or" (Teodorescu, 2019: 78) et faire des provisions alimentaires tout en respectant les "tickets" (Teodorescu, 2019: 33) de rationnement. Ce personnage se trouve ainsi dans une position complexe et profite de sa salle de bains pour échapper de ce train-train quotidien profondément marqué, en outre, par l'expérience totalitaire. De ce fait, nous pouvons affirmer qu'il s'agit une histoire romancée qui atteint les sentiments des lecteurs féminins car, tout comme le signalent Rosa de Diego et Lydia Vázquez (2002: 38) “quizás las mujeres seamos todas madres. Madres con hijos y con urgencia de que sean siempre unos chiquillos necesitados de protección. Madres a pesar de no tenter hijos, pero con padres, hermanos, amigos, compañeros... que imaginamos dependientes de nosotras".

La multiplication des niveaux de récit, le jeu entre la fiction et le document et la représentation de l'individu en tant que sujet traversé par l'Histoire bâtissent ainsi l'artifice de communication romanesque qui s'étale de Mars 1989 à Janvier, Février 1990. Cette ouverture vers l'année suivante permet de mieux comprendre les événements de 1989 en Roumanie où 'l'histoire officielle intègre la manifestation d'avril-juin 1990 en tant que prolongement de la 'Révolution' de décembre 1989” (Heurtaux \& Pellen, 2009: 149). C'est dans ce contexte que les échos de la chute du mur de Berlin sont formellement contestés par Ema qui, dans ses enregistrements, évoque le sujet comme suit:

chez nous, il n'y a pas de mur. C'est un problème majeur. Quand il y a un mur, tu sais exactement comment t'y prendre, si t'as une pioche, un marteau, des clous, une perceuse, si t'as du courage, c'est simple: tu casses. Bien sûr, un mur en béton n'est pas un château de confettis, il ne tombe pas si tu souffles dessus, il faut y aller de toute ta 
force. Mais chez nous il faudrait casser quoi? Le nouveau boulevard [...]? Son nouveau palais à lui, le Génie des Carpates, qu'il appelle sans vergogne la Maison du Peuple? (Teodorescu, 2019: 150-151).

Il s'agit, en effet, d'une remarque assez intéressante d'autant plus qu'elle met en lumière non seulement une revendication des acteurs collectifs, mais aussi la difficulté existante à détruire ce que le système avait construit dans le pays. Cette réflexion se verra par la suite confirmée lors du renversement du regime Ceausescu au moment des multiples mobilisations multisectiorielles qui marqueront la période de cette "Révolution" de décembre 1989 où "ceux qui marchaient comme lors d'une battue, avaient allumé d'immenses feux de joie en utilisant exclusivement des feuilles déchirées du grand livre Homage [...] L'époque de Nicolae Ceausescu, la brillante affirmation de la Roumanie dans la conscience mondiale" (Teodorescu, 2019: 194). La complexité de ces événements illustraient “à la fois une révolution et un coup d'État, que [Carmen] savait grâce à [son] point de vue distancié; [mais] que les gens [...] originaires du même pays, ne pouvaient toujours pas accepter, tant d'années après la révolution" (Teodorescu, 2019: 166). "Le passé qui ne passe pas" (Heurtaux \& Pellen, 2009: 150) semble, en effet, se ressentir au sein des sociétés est-européennes à travers les xénographies francophones de l'extrême contemporain.

\section{Conclusion}

Au terme de cette brève étude sur le dernier roman de l'auteure franco-roumaine Irina Teodorescu nous pensons avoir pu apporter un éclairage sur la traversée littéraire d'un exemple emblématique de ces écrivains qui, ayant vécu sous le joug des totalitarismes, parsèment la scène des xénographies francophones dans l'Europe de nos jours. Dans ce contexte, il est intéressant de noter que

lorsque le déplacement géographique est engendré par une pression politique, le rap-
port de l'auteur à son passé est beaucoup plus aigu, ce qui l'amène immanquablement
à s'interroger sur la reconstruction d'une existence sur les ruines d'un passé endeuillé,
ainsi que sur la bipolarité de son identité. L'impact de cet état ambivalent conditionne
l'écriture produite dans un ailleurs contraint. [...] L'ailleurs est alors ressenti comme
une ouverture, comme la possibilité de découvrir un autre monde, sans renoncer à son
identité première (Enderlein \& Mihova, 2013: 19).

Le tissu fictionnel devient ainsi un rituel de passage où l'écrivain, passeur ou errant, introduit une réflexion identitaire qui permet de penser le rapport non seulement à l'autre, mais aussi aux autres modes de vie. Le panorama littéraire francophone en Europe présente, de ce fait, une mosaïque composée d'éclats de vie d'ici et d'ailleurs qui permettent de mieux comprendre les convulsions d'une page de l'histoire récente. L'expérience fictionnelle de ce corpus d'écrivains est vite remarquée par la critique et même primée, ce qui permet de 
souligner une qualité esthétique indéniable. Tel est le cas d'Irina Teodorescu qui à travers la composition formelle et l'agencement thématique de son parcours romanesque met en musique une production littéraire marquante et reconnue.

En outre, dans cette modernité liquide (Bauman, 2003) où les personnages s'éloignent de la description claire et bien définie, le tissu fictionnel propose une expérience volontairement imprécise. Ni poète ni animal s'inscrit dans ce parcours à travers la construction d'un groupe de personnages que nous pourrions caractériser de liquides, c'est-à-dire "seres borrosos, sin contornos definidos ni caracteres estables, seres sumergidos en sus pensamientos cambiantes, presos del continuo devenir del flujo de la vida" (Montaner, 2013: 413). C'est donc à travers le regard de ces personnages que nous découvrons la portée existentielle de trois femmes dont deux d'entre elles se présentent comme victimes mentales d'un système totalitaire.

L'expérience vécue par l'auteure dans sa Roumanie natale bâtit alors les piliers d'un édifice romanesque à portée autofictionnel. Force est de constater que l'autofiction met en lumière l'expression volontairement imprécise du vécu de l'auteur qui sera par la suite sublimé par l'écriture. Irina Teodorescu se sert alors de ce filtre romanesque qui transforme les épreuves personnelles dans le tissu fictionnel, même si dans son entretien sur France Inter (Varier, 2020: en ligne) elle n'hésite pas à décortiquer les fragments de son parcours biographique présents dans le roman. À ce stade de la réflexion, il est intéressant de signaler l'existence ce corpus d'écrivains transfrontaliers qui, tout comme Irina Teodorescu, s'exposent au grand public à travers l'écriture autofictionnelle. Il s'agit d'un espace qui permet aux auteurs de prendre de la distance avec un sujet parfois douloureux à la fois qu'ils peuvent parsemer l'aventure romanesque avec des éclats de vie d'ici et de là. Cette volonté mémorielle ainsi que le devoir de témoignage présentent sans aucun doute un scénario propice à la réflexion autour des sujets importants de notre histoire récente. C'est dans ce contexte, et en guise de conclusion, que nous pouvons affirmer que les xénographies francophones actuelles exposent ce processus de reconstruction mémorielle d'une génération d'écrivains profondément marquée par l'expérience totalitaire à la fois qu'elle permet de penser et de repenser l'Histoire afin d'éviter le naufrage des civilisations (Maalouf, 2019).

\section{Referencias bibliográficas}

Alfaro, Margarita, Arias, Silvia \& Gamba, Ana. 2019. Agenda 2030. Claves para la transformación sostenible. Madrid, Catarata.

Alfaro, Margarita, Sawas, Stéphane \& Ana Belén Soto. 2020. Xénographies féminines dans l'Europe d'aujourd'hui. Bruxelles, Peter Lang.

Arendt, Hannah. 2002. Le système totalitaire. Les origines du totalitarisme. Paris, Seuil.

Bauman, Zygmunt. 2003. Modernidad líquida. Mexico, Fondo de cultura Económica. 
Anales de Filología Francesa, n. ${ }^{\circ} 28,2020$

EXPÉRIENCE TOTALITAIRE EN EUROPE ET POÉTIQUE DU FÉMININ DANS NI POÈTE NI ANIMAL...

Bogdan, Henry. 1991. Histoire des pays de l'Est. Des origines à nos jours. Paris, Perrin.

Bressiere, Jean. 2011. Littératures d'aujourd'hui: contemporain, innovation, partages culturels, politique, théorie littéraire. Paris, Honoré Champion.

Compagnon, Antoine. 2007. La littérature, pour quoi faire? Paris, Collège de France / Fayard.

De Diego, Rosa \& Vázquez, Lydia. 2002. Figuras de mujer. Madrid, Alianza Editorial.

Delbart, Anne-Rosine. 2015. Les exilés du langage. Un siècle d'écrivains français venus d'ailleurs (1919-2000). Limoges, Pulim.

Delmas-Marty, Mireille. 2016. Aux quatre vents du monde. Petit guide de navigation sur l'océan de la mondialisation. Paris, Seuil.

ENDERLEIn, Évelyne \& Mihova, Lidiya. 2013. Écrire l'ailleurs au féminin dans le monde slave au XXe siècle. Paris, L'Harmattan.

Grell, Isabelle. 2014. L'Autofiction. Paris, Armand Colin.

Hertaux, Jérôme \& Pellen, Cédric. 2009. 1989 à l'Est de l'Europe. Une mémoire controversée. Paris, Éditions de l'aube.

Hubier, Sébastien. 2003. Littératures intimes. Les expressions du moi, de l'autobiographie à l'autofiction. Paris, Armand Colin.

KHAN, Salah. 2017. “'L'hallucination, cet hôte étrange': les limites de la raison au Club des Hachichins" in Çédille, Monografías n 7, 89-108.

Kristeva, Julia. 1988. Étrangers à nous-même. Paris, Gallimard.

Lazarova, Rouja. 1998. Sur le bout de la langue. Paris, Éditions 00h00.

MaAlou, Amin. 2019. Le naufrage des civilisations. Paris, Grasset.

Martínez De SAS, María Teresa. 1999. La lucha por la diversidad en la Europa central y oriental. Barcelone, Ariel Practicum.

Maths-Möser, Ursula \& Mertz-Baumgartner, Birgitz. 2012. Passages et ancrages. Dictionnaire des écrivains migrants de langue française (1981-2011). Paris, Honoré Champion.

Montaner, Lucía. 2013. "Personajes líquidos, personajes íntimos: un estudio sobre el personaje en Le Magot de Momm y La Folie Silaz de Hélène Lenoir" in Çédille, nº 9, 409-421.

Nowicki, Joanna \& Mayaux, Catherine. 2012. L'Autre Francophonie. Paris, Honoré Champion.

ObergöKer, Timo. 2011. Les lieux de l'extrême contemporain. Munich, Martin Meidenbauer.

Plante, Christine. 2003. "La place des femmes dans l'histoire littéraire: annexe ou point de départ d'une relecture critique" in Revue d'histoire littéraire de la France, vol. 3, 655-668. 
Anales de Filología Francesa, n. ${ }^{\circ} 28,2020$

Ana Belén Soto

Porra, Véronique. 2011. Langue française, langue d'adoption. Une littérature "invitée", entre création, stratégies et contraintes (1946-2000). Hildesheum/Zürich/New York, Olms.

Soulet, Jean-François. 2006. Histoire de l'Europe de l'Est de la Seconde Guerre mondiale à nos jours. Paris, Armand Colin.

Teodorescu, Irina. 2019. Ni poète ni animal. Paris, Flammarion.

Teodorescu, Irina. 2018. Celui qui comptait être heureux longtemps. Paris, Gaïa.

Teodorescu, Irina. 2015. Les étrangères. Paris, Gaïa.

Teodorescu, Irina. 2014. La malédiction du bandit moustachu. Paris, Gaïa.

Teodorescu, Irina. 2011. Treize . Paris, EMUE.

Todorov, Tveztan. 2009. L'expérience totalitaire. Paris, Seuil.

Todorov, Tveztan. 2007. La littérature en péril. Paris, Flammarion.

Todorov, Tveztan. 2004. Les abus de la mémoire. Paris, Arléa.

Todorov, Tveztan. 1996. L’homme dépaysé. Paris, Seuil.

Todorov, Tveztan. 1991. Face à l'extrême. Paris, Seuil.

VARIER, Zoé. 2020. “Irina Teodorescu, écrivaine: 'On m'a dit de rester couchée pour éviter les tirs"' in Une journée particulière. Paris, FranceInter. <https://www.franceinter.fr/emissions/une-journee-particuliere/une-journee-particuliere-16-fevrier-2020> [12/05/2020]. 
